\title{
Input Disturbance Rejection in Channel Signal-to-Noise Ratio Constrained Feedback Control
}

\author{
A.J. Rojas, R.H. Middleton, J.S. Freudenberg and J.H. Braslavsky
}

\begin{abstract}
Communication channels impose a number of obstacles to feedback control. One recent line of work considers the problem of feedback stabilisation subject to a constraint on the channel signal-to-noise ratio (SNR). It has been shown for continuous-time systems that the optimal control problem of achieving the infimal SNR can be formulated as a linear quadratic Gaussian (LQG) control problem with weights chosen as in the loop transfer recovery (LTR) technique. The present paper extends this formulation to: discretetime systems; communications over channels with memory; and input disturbance rejection. By using this formulation, we derive exact expressions for the linear time invariant (LTI) controller that achieves the infimal SNR under the effect of time-delay and additive coloured noise. We then quantify the infimal SNR required for both stabilisation and input disturbance rejection for a relative degree one, minimum phase plant and a memoryless Gaussian channel.
\end{abstract}

\section{INTRODUCTION}

The study of control problems with feedback over communication channels has grown rapidly in recent years; see for example [1], [2] and references therein. Communication channels impose additional limitations to feedback, such as constraints in transmission data rate and bandwidth, and effects of noise and time-delay.

Recent developments for performance in the presence of stochastic input disturbance include [3], where an extension of the well-known Bode Integral is achieved by information theoretic arguments; and [4], where a gain pre- and postcompensator is proposed to satisfy a simultaneous power constraint at the plant output and at the input of a memoryless AWGN feedback channel.

The present paper extends the SNR framework in [5] to the case in which input disturbance rejection properties are required in addition to closed-loop stability. We consider a discrete-time feedback system as shown in Figure 1 with an ACGN channel with memory on the measurement path. The plant is assumed to be unstable and possibly include non-minimum phase zeros and time delay. Input disturbances are incorporated in the SNR framework by posing the SNR-constrained feedback problem as an LQG/LTR

March 26, 2008. This work was supported by the ARC Centre of Excellence for Complex Dynamic Systems and Control, 2308, Australia.

A.J. Rojas and J.H. Braslavsky are with the ARC Centre of Excellence for Complex Dynamic Systems and Control, School of Electrical Engineering and Computer Science, The University of Newcastle, 2308, Australia. Emails: alejandro.rojas@newcastle.edu.au, julio.braslavsky@newcastle.edu.au

R.H. Middleton is with the Hamilton Institute, National University of Ireland Maynooth, Co Kildare, Ireland. Email: richard.middleton@nuim.ie

J.S. Freudenberg is with the Department of Electrical Engineering and Computer Science, University of Michigan, Ann Arbor, MI, 48109-2122, U.S.A. Email: jfr@eecs.umich.edu

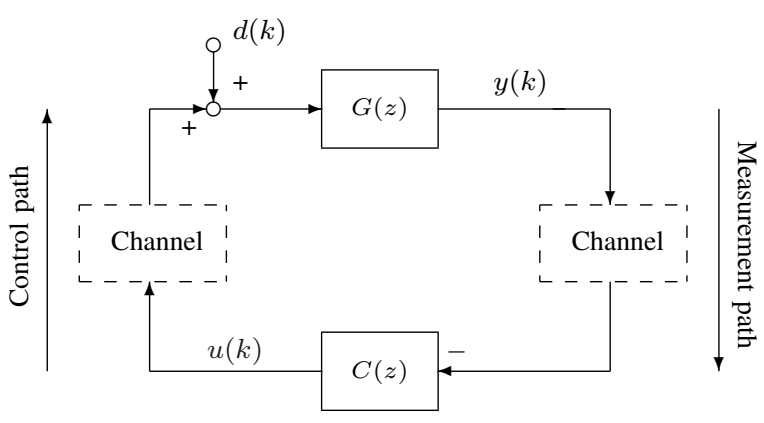

Fig. 1. General problem setting.

optimisation. The main result of the present paper gives the optimal filtering [6] one-degree-of-freedom LTI controller that achieve the infimal SNR for input disturbance rejection with stability. We characterise our optimal solution in terms of a spectral factorisation, which we apply to obtain a closed-form expression of the infimal SNR for disturbance rejection with stability for the case of AWGN channels.

The obtained result extend on [4], when the pre- and postcompensator gain is set to 1 , since the memoryless AWGN channel model is a special case of the ACGN channel with memory model. In comparison to [3] our contribution is that, although restricted to an LTI framework, we obtain exact expressions for the optimal controller achieving the fundamental limitation represented by the infimal SNR for stabilisability and input disturbance rejection.

The rest of the paper is organized as follows: Section II introduces some preliminary concepts and the problem definition. Section III presents the LQG/LTR at the output solution for the closed-loop stabilisability and input disturbance rejection problem when an ACGN channel with memory is located between the plant and the controller. Section IV introduces a spectral factorisation analysis of the LQG/LTR at the output solution. In Section V, with the insight provided by the spectral factorisation argument, we proceed to quantify the infimal LTI SNR required for stabilisability and input disturbance rejection for the case of a minimum phase plant with relative degree one and a memoryless AWGN channel. Finally Section VI presents the conclusions and final remarks for the present work.

Terminology: let $\mathbb{C}$ denote the complex plane. Let $\mathbb{D}^{-}$, $\overline{\mathbb{D}}^{-}, \mathbb{D}^{+}$and $\overline{\mathbb{D}}^{+}$denote respectively the open unit-disk, closed unit-disk, and their complements in the complex plane $\mathbb{C}$. A discrete-time signal is denoted by $x(k), k=$ $0,1,2, \cdots$, and its $\mathcal{Z}$-transform by $X(z), z \in \mathbb{C}$. The expectation operator is denoted by $\mathcal{E}$. A rational transfer 


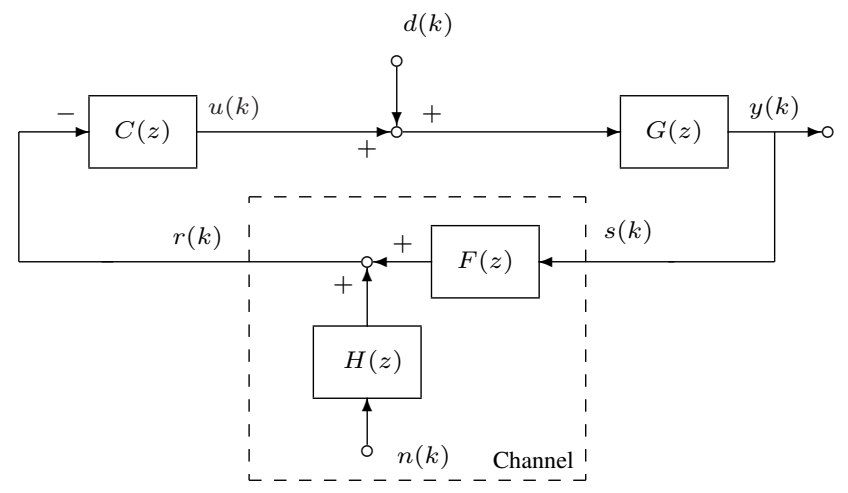

Fig. 2. Output feedback control stabilisation of a discrete-time unstable plant subject to input disturbance over a discrete-time ACGN channel with memory.

function of a discrete-time system is minimum phase if all its zeros lie in $\overline{\mathbb{D}}^{-}$, and is non minimum phase if it has zeros in $\mathbb{D}^{+}$. Given $P(z)$, the transfer function of a discretetime system, we say that $P(z) \in H_{2}$ if $P(z)$ is proper and stable; i.e, relative degree greater than zero all its poles lie in $\mathbb{D}^{-}$. The squared $H_{2}$ norm of $P(z)$, denoted by $\|P\|_{H_{2}}^{2}$, is $\|P\|_{H_{2}}^{2}=(1 / 2 \pi) \int_{-\pi}^{\pi}\left|P\left(e^{j \omega}\right)\right|^{2} d \omega$. If $a$ in $\mathbb{C}, \bar{a}$ represents its complex conjugate.

\section{ASSUMPTIONS AND PROBLEM DEFINITION}

We consider the discrete-time feedback system depicted in Figure 2. The ACGN channel with memory is characterized by two transfer functions, $F(z)$ and $H(z)$, and two parameters: the admissible input power level of the channel, $\mathcal{P}$, and the zero-mean i.i.d. Gaussian white noise $n(k)$ with variance $\sigma^{2}$

\section{A. Assumptions}

General assumptions for the LTI filters in Figure 2, which will be in place unless stated otherwise, are

Plant model:throughout the present work it is assumed that the plant model $G(z)$ is detectable and stabilisable. It is also assumed that $G(z)$ is minimum phase, has $m$ unstable poles, $\left|\rho_{l}\right|>1, \forall l=1, \cdots, m$, and relative degree $n_{g} \geq 1$. Matrices $\left(\mathbf{A}_{\mathbf{G}}, \mathbf{B}_{\mathbf{G}}, \mathbf{C}_{\mathbf{G}}\right)$ represent a minimal realisation of $G(z)$.

Channel model: the channel model $F(z)$ is a stable, minimum phase, biproper transfer function. Matrices $\left(\mathbf{A}_{\mathbf{F}}, \mathbf{B}_{\mathbf{F}}, \mathbf{C}_{\mathbf{F}}, \mathbf{D}_{\mathbf{F}}\right)$ represent a minimal realisation of $F(z)$.

Channel additive noise process: the channel additive noise process is labelled $n(k)$ and it is a zero-mean i.i.d. Gaussian white noise process with variance $\sigma^{2}$.

Noise model: the system $H(z)$ colouring the channel additive white noise $n(k)$ is assumed to be a stable, biproper and minimum phase transfer function. Matrices $\left(\mathbf{A}_{\mathbf{H}}, \mathbf{B}_{\mathbf{H}}, \mathbf{C}_{\mathbf{H}}, \mathbf{D}_{\mathbf{H}}\right)$ represent a minimal realisation of $H(z)$.
Input disturbance process: the input disturbance process is labelled $d(k)$ and it is a zero-mean i.i.d. Gaussian white noise process with variance $\sigma_{d}^{2}$.

Notice that if we lift the assumption of $G(z)$ minimum phase, then we would be required to invoke an inner factorisation argument similar to the one presented in [7], [8]. This would result in terms $C_{\zeta}(z)$ and $\mathbf{C}_{\mathbf{m}}$, where

$$
G(z)=C_{\zeta}(z) \mathbf{C}_{\mathbf{m}}\left(z \mathbf{I}-\mathbf{A}_{\mathbf{G}}\right)^{-1} \mathbf{B}_{\mathbf{G}}
$$

and $C_{\zeta}(z)$ is an all-pass function containing the NMP zeros of $G(z)$ (for more details see also [9, pp. 144-145]). On the other hand, if we lift the minimum phase assumption on $F(z)$, then we could define $F(z)=B_{\zeta F}(z) \tilde{F}(z)$, with $B_{\zeta F}(z)$ an all-pass function containing the NMP zeros of $F(z)$. If we include the term $B_{\zeta F}(z)$ into the plant model as $\tilde{G}(z)=B_{\zeta F}(z) G(z)$, we would then consider the setting in Figure 2 for $(\tilde{G}(z), \tilde{F}(z), H(z))$ instead of the original $(G(z), F(z), H(z))$. Since $B_{\zeta F}(z)$ is all-pass it will not modify the power constraint at the channel input. Finally, the minimum phase assumption for $H(z)$ is without loss of generality, since any NMP zero in $H(z)$ can be factored into an all-pass function, similar to $B_{\zeta F}(z)$, and dropped from the analysis.

\section{B. Problem Definition}

As already mentioned in the introduction, we wish to guarantee the internal stability of the control feedback loop, as well as optimally reject input disturbances when explicitly considering an ACGN channel with memory. We assume that $C(z)$ is such that the closed-loop system is stable in the sense that, for any distribution of initial conditions, the distribution of all signals in the loop will converge exponentially rapidly to a stationary distribution. The channel input power, defined by $\|s\|_{\text {Pow }} \triangleq \lim _{k \rightarrow \infty} \mathcal{E}\left\{y^{2}(k)\right\}$ is required to satisfy an imposed power constraint

$$
\mathcal{P}>\mathcal{E}\left\{y^{2}\right\}
$$

for some predetermined power level $\mathcal{P}$, where $\mathcal{E}\left\{y^{2}\right\}$ stands for $\lim _{k \rightarrow \infty} \mathcal{E}\left\{y^{2}(k)\right\}$ and it is introduced to simplify the notation. Under reasonable stationarity assumptions [10, 44.4$]$, the power in the channel input may be computed as

$$
\mathcal{E}\left\{y^{2}\right\}=\frac{1}{2 \pi} \int_{-\pi}^{\pi}\left|T_{y n}\left(e^{j \omega}\right)\right|^{2} \sigma^{2} d \omega+\frac{1}{2 \pi} \int_{-\pi}^{\pi}\left|T_{y d}\left(e^{j \omega}\right)\right|^{2} \sigma_{d}^{2} d \omega,
$$

where

$$
\begin{aligned}
& T_{y n}(z)=-\frac{C(z) G(z)}{1+C(z) G(z) F(z)} H(z), \\
& T_{y d}(z)=\frac{G(z)}{1+C(z) G(z) F(z)},
\end{aligned}
$$

are the transfer functions that relate $y(k)$ with $n(k)$ and $d(k)$. Since the feedback control system is stable, we have

$$
\mathcal{E}\left\{y^{2}\right\}=\left\|T_{y n}(z)\right\|_{H_{2}}^{2} \sigma^{2}+\left\|T_{y d}(z)\right\|_{H_{2}}^{2} \sigma_{d}^{2}
$$


Thus, the power constraint (2) at the input of the channel translates to the SNR bound on the $H_{2}$ norms of $T_{y n}(z)$ and $T_{y d}(z)$

$$
\frac{\mathcal{P}}{\sigma^{2}}>\left\|T_{y n}(z)\right\|_{H_{2}}^{2}+\left\|T_{y d}(z)\right\|_{H_{2}}^{2} \frac{\sigma_{d}^{2}}{\sigma^{2}} .
$$

Remark 1: It can be seen from (3) and (4) that the biproper assumption for $F(z)$ and $H(z)$ is without loss of generality. Indeed if the transfer function $F(z)$ has relative degree $n_{f}$, with $n_{f} \geq 1$, then we can observe from equation (4) that the case of $F(z)$ strictly proper would be equivalent to

$$
F_{b i p}(z)=z^{n_{f}} F(z), \quad \tilde{G}(z)=\frac{G(z)}{z^{n_{f}}},
$$

since the factor $z^{-n_{f}}$ would not modify the $H_{2}$ norms of $T_{y n}(z)$ or $T_{y d}(z)$. Similarly, if the transfer function $H(z)$ has relative degree $n_{h}$, with $n_{h} \geq 1$, we can observe from equation (4) that this would be equivalent to

$$
H_{b i p}(z)=z^{n} h H(z),
$$

since the factor $z^{n_{h}}$ will not modify the $H_{2}$ norm of $T_{y n}$.

From (4) we observe that a fundamental limitation in the SNR of the ACGN channel will be given by the infimum of $\left\|T_{y n}(z)\right\|_{H_{2}}^{2}$ and $\left\|T_{y d}(z)\right\|_{H_{2}}^{2}$, which indeed are at the core of the infimal LTI SNR problem definition that follows.

Problem 1: (Infimal LTI SNR for Stabilisability and Input Disturbance Rejection LTI Problem). Find $a$ proper rational stabilizing LTI controller $C(z)$ such that the feedback control loop is stable and the transfer functions in (3) achieve the infimum possible constraint (4) imposed on the admissible channel SNR.

The search for the infimal norm of $T_{y n}(z)$ and $T_{y d}(z)$ can be performed in many ways, for example via a Youla parameterisation of all-stabilizing controllers, or by means of LQG estimation with LTR at the output. In the present article we take the second approach since we consider it to be more transparent and less involved when dealing with Problem 1.

\section{LQG/LTR AT THE OUtPut Solution}

From Figure 2 we have that when the ACGN channel with memory is located in the feedback path, the augmented system consisting of $G(z), F(z)$ and $H(z)$ is described by

$$
\begin{aligned}
& x(k+1)=\left[\begin{array}{c}
x^{G}(k+1) \\
x^{F}(k+1) \\
x^{H}(k+1)
\end{array}\right]=\underbrace{\left[\begin{array}{ccc}
\mathbf{A}_{\mathbf{G}} & 0 & 0 \\
\mathbf{B}_{\mathbf{F}} \mathbf{C}_{\mathbf{G}} & \mathbf{A}_{\mathbf{F}} & 0 \\
0 & 0 & \mathbf{A}_{\mathbf{H}}
\end{array}\right]}_{\mathbf{A}_{\mathbf{o}}} x(k)+ \\
& \underbrace{\left[\begin{array}{c}
\mathbf{B}_{\mathbf{G}} \\
0 \\
0
\end{array}\right]}_{\mathbf{B}_{\mathbf{o}}} u(k)+\underbrace{\left[\begin{array}{cc}
\mathbf{B}_{\mathbf{G}} & 0 \\
0 & 0 \\
0 & \mathbf{B}_{\mathbf{H}}
\end{array}\right]}_{\mathbf{E}_{\mathbf{o}}}\left[\begin{array}{l}
d(k) \\
n(k)
\end{array}\right], \\
& r(k)=\underbrace{\left[\begin{array}{lll}
\mathbf{D}_{\mathbf{F}} \mathbf{C}_{\mathbf{G}} & \mathbf{C}_{\mathbf{F}} & \mathbf{C}_{\mathbf{H}}
\end{array}\right]}_{\mathbf{C}_{\mathbf{o}}} x(k)+\underbrace{\left[\begin{array}{ll}
0 & \mathbf{D}_{\mathbf{H}}
\end{array}\right]}_{\mathbf{H}_{\mathbf{o}}}\left[\begin{array}{c}
d(k) \\
n(k)
\end{array}\right] .
\end{aligned}
$$

We assume that the above augmented system is both stabilisable and detectable. Notice that if the plant has relative degree $n_{g}$ then also the overall augmented system $\left(\mathbf{A}_{\mathbf{o}}, \mathbf{B}_{\mathbf{o}}, \mathbf{C}_{\mathbf{o}}, 0\right)$ has relative degree $n_{g}$. In regards to (5), we also have the fact that the measurement and process noises are given by

$$
w(k)=\mathbf{E}_{\mathbf{o}}\left[\begin{array}{c}
d(k) \\
n(k)
\end{array}\right] \quad v(k)=\mathbf{H}_{\mathbf{o}}\left[\begin{array}{c}
d(k) \\
n(k)
\end{array}\right],
$$

and are correlated, thus the covariance matrix is given by

$$
\begin{aligned}
& \mathcal{E}\left\{\left[\begin{array}{c}
w(k) \\
v(k)
\end{array}\right]\left[\begin{array}{ll}
w(j)^{T} & v(j)^{T}
\end{array}\right]\right\}= \\
& \underbrace{\left[\begin{array}{|cc}
\mathbf{o}_{\mathbf{o}}\left[\begin{array}{cc}
\sigma_{d}^{2} & 0 \\
0 & \sigma^{2}
\end{array}\right] \mathbf{E}_{\mathbf{o}}{ }^{T} & \mathbf{E}_{\mathbf{o}}\left[\begin{array}{cc}
\sigma_{d}^{2} & 0 \\
0 & \sigma^{2}
\end{array}\right] \mathbf{H}_{\mathbf{o}}{ }^{T} \\
\mathbf{H}_{\mathbf{o}}\left[\begin{array}{cc}
\sigma_{d}^{2} & 0 \\
0 & \sigma^{2}
\end{array}\right] \mathbf{E}_{\mathbf{o}}{ }^{T} & \mathbf{H}_{\mathbf{o}}\left[\begin{array}{cc}
\sigma_{d}^{2} & 0 \\
0 & \sigma^{2}
\end{array}\right] \mathbf{H}_{\mathbf{o}}{ }^{T}
\end{array}\right]}_{\left[\begin{array}{cc}
\mathbf{W} & \mathbf{S} \\
\mathbf{S}^{T} & \mathbf{V}
\end{array}\right]} \delta_{i j},
\end{aligned}
$$

with $\delta_{i j}$ the Kronecker's delta. By replacing $\mathbf{E}_{\mathbf{o}}$ and $\mathbf{H}_{\mathbf{o}}$ we obtain

$$
\begin{aligned}
\mathbf{W} & =\left[\begin{array}{ccc}
\mathbf{B}_{\mathbf{G}} \mathbf{B}_{\mathbf{G}^{T}} \sigma_{d}^{2} & 0 & 0 \\
0 & 0 & 0 \\
0 & 0 & \mathbf{B}_{\mathbf{H}} \mathbf{B}_{\mathbf{H}}^{T} \sigma^{2}
\end{array}\right], \\
\mathbf{V} & =\mathbf{D}_{\mathbf{H}} \mathbf{D}_{\mathbf{H}}^{T} \sigma^{2}, \\
\mathbf{S} & =\left[\begin{array}{c}
0 \\
0 \\
\mathbf{B}_{\mathbf{H}} \mathbf{D}_{\mathbf{H}}^{T} \sigma^{2}
\end{array}\right] .
\end{aligned}
$$

In general the procedure of LQG optimisation with recovery involves the solution of two Riccati equations, one associated with the design of the observer and another with the design of the regulator. For the LQG/LTR procedure at the output the regulator's Riccati equation is dictated by the LTR procedure; whilst the observer's Riccati equation is a function of user/design requirements. In our case, this is based around a minimum energy design, since we want to infimise the channel input variance.

The Riccati equations for both the regulator and observer (and associated gains) are listed below. For the observer

$$
\begin{aligned}
\boldsymbol{\Sigma}_{\mathbf{o}}= & \mathbf{A}_{\mathbf{o}} \boldsymbol{\Sigma}_{\mathbf{o}} \mathbf{A}_{\mathbf{o}}{ }^{T}-\left(\mathbf{A}_{\mathbf{o}} \boldsymbol{\Sigma}_{\mathbf{o}} \mathbf{C}_{\mathbf{o}}{ }^{T}+\mathbf{S}\right) \\
& \left(\mathbf{C}_{\mathbf{o}} \boldsymbol{\Sigma}_{\mathbf{o}} \mathbf{C}_{\mathbf{o}}{ }^{T}+\mathbf{V}\right)^{-1}\left(\mathbf{C}_{\mathbf{o}} \boldsymbol{\Sigma}_{\mathbf{o}} \mathbf{A}_{\mathbf{o}}{ }^{T}+\mathbf{S}^{T}\right)+\mathbf{W}^{T}, \\
\mathbf{K}_{\mathbf{p o}}= & \mathbf{A}_{\mathbf{o}} \boldsymbol{\Sigma}_{\mathbf{o}} \mathbf{C}_{\mathbf{o}}{ }^{T}\left(\mathbf{C}_{\mathbf{o}} \boldsymbol{\Sigma}_{\mathbf{o}} \mathbf{C}_{\mathbf{o}}{ }^{T}+\mathbf{V}\right)^{-1},
\end{aligned}
$$

and for the regulator

$$
\begin{aligned}
& \mathbf{P}_{\mathbf{o}}=\mathbf{A}_{\mathbf{o}}{ }^{T} \mathbf{P}_{\mathbf{o}} \mathbf{A}_{\mathbf{o}}-\mathbf{A}_{\mathbf{o}}{ }^{T} \mathbf{P}_{\mathbf{o}} \mathbf{B}_{\mathbf{o}}\left(\mathbf{B}_{\mathbf{o}}{ }^{T} \mathbf{P}_{\mathbf{o}} \mathbf{B}_{\mathbf{o}}\right)^{-1} \mathbf{B}_{\mathbf{o}}{ }^{T} \mathbf{P}_{\mathbf{o}} \mathbf{A}_{\mathbf{o}}, \\
& \mathbf{K}_{\mathbf{o}}=\left(\mathbf{B}_{\mathbf{o}}{ }^{T} \mathbf{P}_{\mathbf{o}} \mathbf{B}_{\mathbf{o}}\right)^{-1} \mathbf{B}_{\mathbf{o}}{ }^{T} \mathbf{P}_{\mathbf{o}} \mathbf{A}_{\mathbf{o}} .
\end{aligned}
$$

As a result of the fact that the design of the observer is predefined in the recovery at the output procedure, we have that the regulator gain has a specific closed-form solution [8, Theorem 2.1]

$$
\mathbf{K}_{\mathbf{o}}=\left(\mathbf{C}_{\mathbf{G}} \mathbf{A}_{\mathbf{G}^{n}}{ }^{n^{-1}} \mathbf{B}_{\mathbf{G}}\right)^{-1}\left[\begin{array}{lll}
\mathbf{C}_{\mathbf{G}} & 0 & 0
\end{array}\right] \mathbf{A}_{\mathbf{o}}{ }^{{ }^{n} g} .
$$

Notice that the expression in (10) for the observer gain is correct for minimum phase plants, whilst for non minimum phase (NMP) plants an inner factorisation argument similar 
to the one presented in [8] is required. The reader is referred to [7], [8], [5] and also [9, p.145] for the procedure required in the case of NMP zeros. A general discrete-time observer for the system in (5) is defined by

$$
\begin{array}{r}
\hat{x}(k+1 \mid k)=\mathbf{A}_{\mathbf{o}} \hat{x}(k \mid k-1)+\mathbf{B}_{\mathbf{o}} u(k)+ \\
\mathbf{K}_{\mathbf{p o}}\left(y_{m}(k)-\mathbf{C}_{\mathbf{o}} \hat{x}(k \mid k-1)\right),
\end{array}
$$

where the gain $\mathbf{K}_{\text {po }}$ is chosen such that the matrix $\mathbf{A}_{\mathbf{o}}-$ $\mathbf{K}_{\mathbf{p o}} \mathbf{C}_{\mathbf{o}}$ has stable eigenvalues. In Figure 2 the measured output is $r(k)$.

A filtering observer is defined by (11) with $\mathbf{K}_{\text {po }}=$

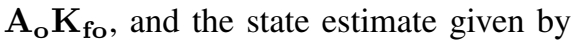

$$
\hat{x}(k \mid k)=\hat{x}(k \mid k-1)+\mathbf{K}_{\mathbf{f o}}\left(y_{m}(k)-\mathbf{C}_{\mathbf{o}} \hat{x}(k \mid k-1)\right) .
$$

If the filtering observer is considered together with the linear control law

$$
u(k)=-\mathbf{K}_{\mathbf{o}} \hat{x}(k \mid k),
$$

we obtain a filtering observer-based compensator given by

$$
C_{f}(z)=z \mathbf{K}_{\mathbf{o}}\left(z \mathbf{I}-\left(\mathbf{I}-\mathbf{K}_{\mathbf{f o}} \mathbf{C}_{\mathbf{o}}\right)\left(\mathbf{A}_{\mathbf{o}}-\mathbf{B}_{\mathbf{o}} \mathbf{K}_{\mathbf{o}}\right)\right)^{-1} \mathbf{K}_{\mathbf{f o}} .
$$

In particular the gain $\mathbf{K}_{\mathbf{o}}$ for the linear control law is tuned to achieve cheap control as in [8, Theorem 2.1]. Define the observer open loop transfer function and sensitivity function as

$$
L_{e s t}(z)=\mathbf{C}_{\mathbf{o}} \Phi_{\mathbf{o}}(z) \mathbf{K}_{\mathbf{p o}}, \quad S_{e s t}(z)=\left(1+L_{e s t}(z)\right)^{-1},
$$

where $\Phi_{\mathbf{o}}(z)=\left(z I-\mathbf{A}_{\mathbf{o}}\right)^{-1}$. Define also the open loop transfer function and sensitivity function as

$$
L(z)=C_{f}(z) G(z) F(z), \quad S(z)=(1+L(z))^{-1} .
$$

The overall goal is to obtain a transfer function $E_{d o}(z)$ such that

$$
S(z)=\left(1+E_{d o}(z)\right) S_{e s t}(z) .
$$

As a matter of notation, we specify the observer gain $\mathbf{K}_{\mathbf{p o}}$ in (8) to be

$$
\mathbf{K}_{\mathbf{p o}}=\left[\begin{array}{lll}
\left(\mathbf{K}_{\mathbf{p o}}^{\mathbf{g}}\right)^{T} & \left(\mathbf{K}_{\mathbf{p o}}^{\mathbf{f}}\right)^{T} & \left(\mathbf{K}_{\mathbf{p o}}^{\mathbf{h}}\right)^{T}
\end{array}\right]^{T},
$$

where the dimension of each component is compatible with the components of the state vector $x(k)$ in (5).

Theorem 1: (Optimal LTI Controller for Stabilisation and Input Disturbance Rejection) Take the system with feedback over an ACGN channel with memory as shown in Figure 2. Let $\mathbf{K}_{\mathrm{po}}$ be the optimal observer gain obtained from (8) with $\mathbf{A}_{\mathbf{o}}, \mathbf{B}_{\mathbf{o}}, \mathbf{C}_{\mathbf{o}}, \mathbf{W}, \mathbf{V}, \mathbf{S}$ as defined for the augmented plant/channel structure given by (5) and (7).

Then the infimal LTI SNR for stabilisability and input disturbance rejection LTI problem is solved by the "filtering" controller

$$
\begin{aligned}
C_{f}(z)=\left(\mathbf{C}_{\mathbf{G}} \mathbf{A}_{\mathbf{G}}{ }^{n_{g}-1}\right. & \left.\Phi_{\mathbf{G}} \mathbf{B}_{\mathbf{G}}\right)^{-1} \\
& \left(\mathbf{C}_{\mathbf{G}} \Phi_{\mathbf{G}} \mathbf{A}_{\mathbf{G}}{ }^{n-1} \mathbf{K}_{\mathbf{p o}}^{\mathbf{g}}\right)\left(1+E_{d o}(z)\right)^{-1},
\end{aligned}
$$

where

$$
\begin{aligned}
& E_{d o}(z)=\mathbf{C}_{\mathbf{F}} \Phi_{\mathbf{F}}(z) \mathbf{K}_{\mathbf{p o}}^{\mathbf{f}}+\mathbf{C}_{\mathbf{H}} \Phi_{\mathbf{H}}(z) \mathbf{K}_{\mathbf{p o}}^{\mathbf{h}}+ \\
& F(z)\left(\mathbf{C}_{\mathbf{G}}-\frac{1}{z^{n}{ }^{n-1}} \mathbf{C}_{\mathbf{G}} \mathbf{A}_{\mathbf{G}}{ }^{n_{g}-1}\right) \Phi_{\mathbf{G}}(z) \mathbf{K}_{\mathbf{p o}}^{\mathbf{g}},
\end{aligned}
$$

with $\Phi_{\mathbf{G}}(z)=\left(z \mathbf{I}-\mathbf{A}_{\mathbf{G}}\right)^{-1}, \Phi_{\mathbf{F}}(z)=\left(z \mathbf{I}-\mathbf{A}_{\mathbf{F}}\right)^{-1}, \Phi_{\mathbf{H}}(z)=\left(z \mathbf{I}-\mathbf{A}_{\mathbf{H}}\right)^{-1}$.

Proof: The proof follows the same steps as in the proof of [5, Theorem 2]. The difference with the result in [5] lies in the Riccati equation for the estimator gain $\mathbf{K}_{\mathbf{p o}}$ which in the present paper includes an input disturbance process.

The result of Theorem 1 characterises the controller achieving the infimal LTI SNR for stabilisability and input disturbance rejection. In the next corollary we consider the relative degree of the plant $n_{g}$ to be 1 .

Corollary 1: (LQG/LTR at the Output Characterisation of $T_{y n}(z)$ and $\left.T_{y d}(z)\right)$ Consider the case where the plant relative degree $n_{g}$ is 1 , then

$$
\begin{aligned}
& T_{y n}(z)=-\frac{\mathbf{C}_{\mathbf{G}^{\Phi}} \Phi_{\mathbf{G}}(z) \mathbf{K}_{\mathbf{p o}}^{\mathbf{g}}}{1+\mathbf{C}_{\mathbf{o}} \Phi_{\mathbf{o}}(z) \mathbf{K}_{\mathbf{p o}}} H(z), \\
& T_{y d}(z)=\frac{1+\mathbf{C}_{\mathbf{F}} \Phi_{\mathbf{F}}(z) \mathbf{K}_{\mathbf{p o}}^{\mathbf{f}}+\mathbf{C}_{\mathbf{H}} \Phi_{\mathbf{H}}(z) \mathbf{K}_{\mathbf{p o}}^{\mathbf{h}}}{1+\mathbf{C}_{\mathbf{o}} \Phi_{\mathbf{o}}(z) \mathbf{K}_{\mathbf{p o}}} G(z),
\end{aligned}
$$

Proof: Recall from (15) that $\left(1+E_{d o}(z)\right)^{-1}=$ $S_{\text {est }}(z) / S(z)$ and from (14) that $S_{\text {est }}=1 /\left(1+\mathbf{C}_{\mathbf{o}} \Phi_{\mathbf{o}} \mathbf{K}_{\mathbf{p o}}\right)$. Replace in Theorem 1 together with $n_{g}=1$.

It is well known, when using the LQG/LTR approach for the design of a filtering controller, that if the plant is minimum phase with relative degree 1 we recover the design of the observer, i.e. $S(z)=S_{\text {est }}(z)$. Nonetheless, from $T_{y d}(z)$ in (19) in the above corollary, we have that $E_{d o}(z) \neq 0$. The reason for this stems from the fact that the measured output is not the performance output. Namely, we are observing $r(k)$, but have defined Problem 1 in terms of $y(k)$.

\section{SPECTRAL FACTORISATION}

In the present section we present the spectral factorisation induced by the Riccati equation in (8). The interest in such a spectral factorisation comes from the fact that the observer's design is central to the infimal LTI SNR for stabilisability and input disturbance rejection solution. Thus, by means of a spectral factorisation argument we characterise the optimal $S_{\text {est }}(z)$ that takes part into the infimal LTI SNR for stabilisability and input disturbance rejection solution.

Theorem 2: (Induced Spectral Factorisation) The Riccati equation in (8), with $\mathbf{K}_{\text {po }}$ the optimal observer gain and $\mathbf{A}_{\mathbf{o}}, \mathbf{B}_{\mathbf{o}}, \mathbf{C}_{\mathbf{o}}, \mathbf{W}, \mathbf{V}, \mathbf{S}$ as defined by the augmented plant/channel structure given by (5) and (7), induces the following spectral factorisation

$$
\begin{aligned}
& S_{e s t}^{-1}(z)\left(\mathbf{C}_{\mathbf{o}} \boldsymbol{\Sigma}_{\mathbf{o}} \mathbf{C}_{\mathbf{o}}{ }^{T}+\mathbf{D}_{\mathbf{H}} \mathbf{D}_{\mathbf{H}}^{T} \sigma^{2}\right) S_{e s t}^{-T}\left(z^{-1}\right)= \\
& H(z) \sigma^{2} H\left(z^{-1}\right)+F(z) G(z) \sigma_{d}^{2} G\left(z^{-1}\right) F\left(z^{-1}\right),
\end{aligned}
$$

where $S_{\text {est }}(z)$ is as in (14).

Proof: From equation (5.6) in [11, p. 85], adding $\mathbf{S}$ into the analysis and adapting the notation to the present 
paper, we have

$$
\begin{aligned}
\left(1+\mathbf{C}_{\mathbf{o}}\left(z \mathbf{I}-\mathbf{A}_{\mathbf{o}}\right)^{-1} \mathbf{K}_{\mathbf{p o}}\right)\left(\mathbf{C}_{\mathbf{o}} \boldsymbol{\Sigma}_{\mathbf{o}} \mathbf{C}_{\mathbf{o}}{ }^{T}+\mathbf{V}\right)\left(1+\mathbf{K}_{\mathbf{p o}}{ }^{T}\left(z^{-1} \mathbf{I}-\mathbf{A}_{\mathbf{o}}{ }^{T}\right) \mathbf{C}_{\mathbf{o}}{ }^{T}\right) \\
=\mathbf{V}+\mathbf{C}_{\mathbf{o}}\left(z \mathbf{I}-\mathbf{A}_{\mathbf{o}}\right)^{-1} \mathbf{S}+\mathbf{S}^{T}\left(z^{-1} \mathbf{I}-\mathbf{A}_{\mathbf{o}}{ }^{T}\right)^{-1} \mathbf{C}_{\mathbf{o}}{ }^{T} \\
\quad+\mathbf{C}_{\mathbf{o}}\left(z \mathbf{I}-\mathbf{A}_{\mathbf{o}}\right)^{-1} \mathbf{W}\left(z^{-1} \mathbf{I}-\mathbf{A}_{\mathbf{o}}{ }^{T}\right)^{-1} \mathbf{C}_{\mathbf{o}}{ }^{T}
\end{aligned}
$$

Consider now, to obtain (20), replacing $\mathbf{W}, \mathbf{V}, \mathbf{S}$ as in (7) into (21), $\mathbf{A}_{\mathbf{o}}$ and $\mathbf{C}_{\mathbf{o}}$ as in (5) into the RHS of (21), whilst recognising $S_{\text {est }}(z)$ as in (14) on the LHS of (21), which ends the proof.

From Theorem 2, if we consider the memoryless AWGN channel case we modify our initial assumptions, eliminating from the analysis $F(z)$ and $H(z)$, and observe that the spectral factorisation derived from Theorem 2 becomes

$$
S_{\text {est }}^{-1}(z)\left(\frac{\mathbf{C}_{\mathbf{G}} \mathbf{\Sigma}_{\mathbf{O}} \mathbf{C}_{\mathbf{G}}{ }^{T}}{\sigma^{2}}+1\right) S_{\text {est }}^{-T}\left(z^{-1}\right)=1+G(z) \frac{\sigma_{d}^{2}}{\sigma^{2}} G\left(z^{-1}\right) .
$$

From equation (22) we have that the plant $G(z)$, together with $\sigma^{2}$ and $\sigma_{d}^{2}$, will determine the observer's sensitivity function $S_{\text {est }}(z)$. Notice though that the stable poles of $G(z)$ will also play a role in (22).

\section{QUANTIFYING THE INFIMAL LTI SNR}

\section{REQUIREMENT FOR A MEMORYLESS AWGN CHANNEL}

In the present section we attempt to quantify the infimal LTI SNR for stabilisability and input disturbance rejection. To do so we use the spectral factorisation result in (22) and thus we specify the plant model to be

$$
G(z)=\frac{q(z)}{p(z)}=\frac{q(z)}{\prod_{i=1}^{m}\left(z-\rho_{i}\right)},
$$

where $\rho_{i} \in \mathbb{D}^{+}$, and all $\rho_{i}$ are distinct. The polynomial $q(z)$ is assumed known, with degree $m-1$ and all its solutions in $\mathbb{D}^{-}$. The communication over the measurement path takes place over a memoryless AWGN channel. As we stated before, we are ultimately attempting to characterise the specific $S_{\text {est }}(z)$ that takes part into the infimal LTI SNR for stabilisability and input disturbance rejection solution. Notice then that $S_{\text {est }}(z)$, for the present section choice of plant and channel model, must contain the $m$ unstable plant poles $\rho_{i}$ as NMP zeros to guarantee the internal stability of the closed-loop. Thus, we only have to investigate the location of the poles of $S_{\text {est }}(z)$ for which we use (22) to find that

$$
\begin{aligned}
& S_{\text {est }}^{-1}(z)\left(\frac{\mathbf{C}_{\mathbf{G}} \boldsymbol{\Sigma}_{\mathbf{o}} \mathbf{C}_{\mathbf{G}}{ }^{T}}{\sigma^{2}}+1\right) S_{\text {est }}^{-T}\left(z^{-1}\right)= \\
& \frac{p(z) p\left(z^{-1}\right)+q(z) \frac{\sigma_{d}^{2}}{\sigma^{2}} q\left(z^{-1}\right)}{p(z) p\left(z^{-1}\right)} .
\end{aligned}
$$

From (24) we recognise that the poles of $S_{\text {est }}(z) z_{i}, z_{i} \in$ $\mathbb{D}^{-} i=1, \cdots, m$, are the Schur's solutions of

$$
p(z) p\left(z^{-1}\right)+q(z) \frac{\sigma_{d}^{2}}{\sigma^{2}} q\left(z^{-1}\right)=0,
$$

a polynomial of degree $2 \mathrm{~m}$. It can be shown that the other $m$ solutions of (25) are the reflections of each $z_{i}$, that is $1 / z_{i}, 1 / z_{i} \in \mathbb{D}^{+} i=1, \cdots, m$. We have then that a characterisation of the optimal $S_{\text {est }}(z)$ is given by

$$
S_{\text {est }}(z)=\prod_{i=1}^{m} \frac{z-\rho_{i}}{z-z_{i}} .
$$

We stress that, although we do not have a closed-form for each $z_{i}$, they can be computed by any of the many currently available algorithms for the purpose of finding the solutions of a polynomial, thus for all purposes we consider them as known quantities. Finally notice, also from (25), that as $\sigma_{d}^{2} \rightarrow 0$, each $z_{i}$ will tend to one of the unstable plant poles mirrored images $1 / \rho_{i}$. By means of (26) we are able to quantify the infimal LTI SNR for stabilisability and input disturbance rejection.

Theorem 3: (Infimal LTI SNR for Stabilisability and Input Disturbance Rejection) Assume the plant to be as in (23), the channel model to be a memoryless AWGN channel and the controller to be a filtering controller, then the channel SNR satisfies

$$
\frac{P}{\sigma^{2}}>\sum_{i=1}^{m} \sum_{j=1}^{m} \frac{h_{i} \bar{h}_{j}}{1-z_{i} \bar{z}_{j}}+\frac{\sigma_{d}^{2}}{\sigma^{2}} \sum_{i=1}^{m} \sum_{j=1}^{m} \frac{g_{i} \bar{g}_{j}}{1-z_{i} \bar{z}_{j}},
$$

where

$$
h_{i}=\frac{\prod_{\substack{j=1 \\ j=1 \\ j \neq i}}^{m}\left(z_{i}-\rho_{j}\right)}{\left.\prod_{i}-z_{j}\right)}, \quad \text { and } \quad g_{i}=q\left(z_{i}\right) .
$$

Proof: From Corollary 1 we have that $T_{y n}(z)=-1+$ $S_{\text {est }}(z)$ and $T_{y d}(z)=S_{\text {est }}(z) G(z)$. From (4) we have that the channel SNR satisfies

$$
\frac{P}{\sigma^{2}}>\left\|1-S_{\text {est }}\right\|_{H_{2}}^{2}+\left\|S_{\text {est }} G\right\|_{H_{2}}^{2} \frac{\sigma_{d}^{2}}{\sigma^{2}} .
$$

Replace $S_{\text {est }}(z)$ as in (26) and $G(z)$ as in (23) to obtain

$$
\frac{P}{\sigma^{2}}>\left\|\frac{-\prod_{i=1}^{m}\left(z-z_{i}\right)+\prod_{i=1}^{m}\left(z-\rho_{i}\right)}{\prod_{i=1}^{m=1}\left(z-z_{i}\right)}\right\|_{H_{2}}^{2}+\left\|\frac{q(z)}{\prod_{i=1}^{m}\left(z-z_{i}\right)}\right\|_{H_{2}}^{2} \frac{\sigma_{d}^{2}}{\sigma^{2}} .
$$

A partial fraction expansion leads us to

$$
\frac{P}{\sigma^{2}}>\left\|\sum_{i=1}^{m} \frac{h_{i}}{z-z_{i}}\right\|_{H_{2}}^{2}+\left\|\sum_{i=1}^{m} \frac{g_{i}}{z-z_{i}}\right\|_{H_{2}}^{2} \frac{\sigma_{d}^{2}}{\sigma^{2}}
$$

with $h_{i}$ and $g_{i}$ as in (28). Finally by applying the Residue Theorem, see for example [12, pp. 169-172], we compute both $\mathrm{H}_{2}$ squared norms to obtain the result in (27), which ends the proof.

Remark 2: The assumption in (23) of distinct poles simplifies the partial fraction expansion argument invoked in the proof of Theorem 3, but it is not essential to it.

Theorem 3 quantifies the infimal LTI SNR for stabilisability and input disturbance rejection under the present section assumptions. Notice how if $\sigma_{d}^{2}$ vanishes, then $\frac{\sigma_{d}^{2}}{\sigma^{2}} \sum_{i=1}^{m} \sum_{j=1}^{m} \frac{g_{i} \bar{g}_{j}}{1-z_{i} \bar{z}_{j}}$ vanishes as well, whilst $\sum_{i=1}^{m} \sum_{j=1}^{m} \frac{h_{i} \bar{h}_{j}}{1-z_{i} \bar{z}_{j}}$, through $z_{i} \rightarrow 1 / \rho_{i}$, approaches the known result of $\prod_{i=1}^{m}\left|\rho_{i}\right|^{2}-1$, the infimal LTI SNR for stabilisability [13]. To conclude the discussion developed in the present paper let us introduce a simple example in which we can explicitly account for the pole of $S_{\text {est }}(z)$.

Example 1: Consider the plant to be

$$
G(z)=\frac{K}{z-\rho},
$$

with $\rho \in \mathbb{R}^{+}, \rho>1$ and $K \in \mathbb{R}^{+}$. From (22) we have

$$
\frac{z^{2}+\left(-\rho-\frac{1}{\rho}-\frac{K^{2} \sigma_{d}^{2}}{\rho \sigma^{2}}\right) z+1}{(z-\rho)\left(z-\frac{1}{\rho}\right)},
$$


therefore $z_{1}$, the solution for the numerator and pole of $S_{\text {est }}(z)$, is given by

$$
z_{1}=\frac{\rho+\frac{1}{\rho}+\frac{K \sigma_{d}^{2}}{\rho \sigma^{2}}-\sqrt{\left(-\rho-\frac{1}{\rho}-\frac{K^{2} \sigma_{d}^{2}}{\rho \sigma^{2}}\right)^{2}-4}}{2},
$$

the only solution for the quadratic polynomial $z^{2}+\left(-\rho-\frac{1}{\rho}-\frac{K^{2} \sigma_{d}^{2}}{\rho \sigma^{2}}\right) z+1$ that satisfies ${ }^{1} z_{1} \in \mathbb{D}^{-}$. With $z_{1}$ known, applying Theorem 3 gives

$$
\frac{P}{\sigma^{2}}>\frac{\left(z_{1}-\rho\right)^{2}}{1-z_{1}^{2}}+\frac{\sigma_{d}^{2}}{\sigma^{2}} \frac{K^{2}}{1-z_{1}^{2}},
$$

from which we observe that as $\sigma_{d}^{2} \rightarrow 0$, then $z_{1} \rightarrow 1 / \rho$ and

$$
\frac{P}{\sigma^{2}}>|\rho|^{2}-1,
$$

the infimal LTI SNR for stabilisability result from [13]. Since Theorem 3 is valid for memoryless AWGN channels and since for this type of channels the capacity definition of $C=1 / 2 \log _{2}\left(1+p / \sigma^{2}\right)$ is not increased by the use of feedback, we can also conclude that for $\sigma_{d}^{2} \neq 0$ the channel capacity will exceed the infimal requirement for stabilisability of $1 / 2 \log _{2} \rho$. The extra SNR/Channel capacity requirement might be avoided by explicitly considering encoding and decoding in the communication link since, as for example reported in [14], the optimal LTI solution might not be always the infimal solution.

As a side result we have from $S_{\text {est }}(z)$ as in (26) and the spectral factorisation described in (22) that the innovation covariance $[11, \mathrm{p} .111]$ satisfies

$$
\frac{\mathbf{C}_{\mathbf{G}} \boldsymbol{\Sigma}_{\mathbf{O}} \mathbf{C}_{\mathbf{G}}{ }^{T}}{\sigma^{2}}=\prod_{i=1}^{m} \frac{\rho_{i}}{z_{i}}-1,
$$

which, as $\sigma_{d}^{2} \rightarrow 0$, approaches $\prod_{i=1}^{m}\left|\rho_{i}\right|^{2}-1$ the infimal LTI SNR for stabilisability [13, Theorem III.1].

Finally we stress that the result of Theorem 3 applies to the case of memoryless AWGN channels. It might be feasible to analyse some special cases of channels with coloured noise by means of recent results reported in [15].

\section{CONCLUSION AND REMARKS}

In the present paper we have solved the infimal LTI SNR for stabilisability and input disturbance rejection problem for the case of discrete-time ACGN channels with memory on the measurement path and minimum phase unstable LTI plants with arbitrary relative degree.

By means of a spectral factorisation argument, we have then quantified in a closed-form the infimal LTI SNR required to achieve stabilisability and input disturbance rejection for a class of minimum phase plant models with relative degree one and a memoryless AWGN channel model. We have shown how the obtained SNR approaches known results as the variance of the input disturbance process vanishes. Future directions for research include a more general plant model when quantifying the infimal

${ }^{1}$ This can be seen to be true from the fact that

$$
\rho+\frac{1}{\rho}+\frac{K \sigma_{d}^{2}}{\rho \sigma^{2}}>2
$$

as long as $\rho \in \mathbb{R}^{+}$, which in turns allow us to claim that $z_{1}<1$.
LTI SNR for stabilisability and input disturbance rejection, extending the result to include a filtered input disturbance process, or different injection points for the disturbance process.

\section{ACKNOWLEDGMENTS}

The authors wish to thank the anonymous reviewers for their careful reading and helpful comments.

\section{REFERENCES}

[1] Special Issue on Networked Control Systems. IEEE Transactions on Automatic Control, 49(9), September 2004.

[2] G.N. Nair, F. Fagnani, S. Zampieri, and R.J. Evans. Feedback Control under Data Rate Constraints: an Overview. In Proceedings of the IEEE (special issue on "The Emerging Technology of Networked Control Systems"), January 2007.

[3] N.C. Martins and M.A. Dahleh. Feedback Control in the Presence of Noisy Channels: Bode-Like Fundamental Limitations of Performance. IEEE Transactions on Automatic Control (To appear), 2007. http: //www. glue. umd.edu/ nmartins/bode1.pdf.

[4] J.S. Freudenberg, R.H. Middleton, and J.H. Braslavsky. Stabilization with Disturbance Attenuation over a Gaussian Channel. In Proceedings of the 46th IEEE Conference on Decision and Control, New Orleans, USA, December 2007.

[5] A.J. Rojas, J.S. Freudenberg, J.H. Braslavsky, and R.H. Middleton. Optimal Signal to Noise Ratio in Feedback over Communication Channels with Memory. In Proceedings 45th IEEE Conference on Decision and Control, San Diego, USA, December 2006.

[6] J.M. Maciejowski. Asymptotic Recovery for Discrete-Time Systems. IEEE Transactions on Automatic Control, 30(6):602-605, 1985.

[7] Z. Zhang. Loop Transfer Recovery for Nonminimum Phase Plants and Ill-Conditioned Plants. PhD thesis, The University of Michigan, 1990.

[8] Z. Zhang and J.S. Freudenberg. Discrete-time Loop Transfer Recovery for Systems with Nonminimum Phase Zeros and Time Delays. Automatica, 29(2), March 1993.

[9] K. Zhou, J.C. Doyle, and K. Glover. Robust and optimal control. Prentice Hall, 1996.

[10] K.J. Åström. Introduction to Stochastic Control Theory. Academic Press, 1970.

[11] B. Anderson and J. Moore. Optimal Filtering. Dover publications, NY, 2005.

[12] R.V. Churchill and J.W. Brown. Complex Variables and Applications. McGraw-Hill International Editions, fifth edition, 1990.

[13] J.H. Braslavsky, R.H. Middleton, and J.S. Freudenberg. Feedback Stabilisation over Signal-to-Noise Ratio Constrained Channels. IEEE Transactions on Automatic Control, 52(8):1391-1403, 2007.

[14] N.C. Martins. Witsenhausens Counter Example Holds in the Presence of Side Information. In Proceedings 45th IEEE Conference on Decision and Control, San Diego, USA, 2006.

[15] Y.-H. Kim. Feedback Capacity of the First-Order Moving Average Gausssian Channel. IEEE Transactions on Information Theory, 52(7):pp. 3063-3079, July 2006. 